\title{
New Radiographic Index for Occipito-Cervical Instability
}

\author{
Moon Soo Park ${ }^{1}$, Seong-Hwan Moon ${ }^{2}$, Tae-Hwan $\mathrm{Kim}^{1}$, Jae Keun $\mathrm{Oh}^{3}$, \\ Ji Hoon Nam ${ }^{1}$, Jae Kyun Jung ${ }^{1}$, K. Daniel Riew ${ }^{4}$ \\ ${ }^{1}$ Department of Orthopaedic Surgery, Hallym University Sacred Heart Hospital, Hallym University College of Medicine, Anyang, Korea \\ ${ }^{2}$ Department of Orthopaedic Surgery, Yonsei University College of Medicine, Seoul, Korea \\ ${ }^{3}$ Department of Neurosurgery, Hallym University Sacred Heart Hospital, Hallym University College of Medical, Anyang, Korea \\ ${ }^{4}$ Washington University Orthopedics, BJC Institute of Health at Washington University School of Medicine, Chesterfield, MO, USA
}

\begin{abstract}
Study Design: Retrospective study.
Purpose: To propose a new radiographic index for occipito-cervical instability.

Overview of Literature: Symptomatic atlanto-occipital instability requires the fusion of the atlanto-occipital joint. However, measurements of occipito-cervical translation using the Wiesel-Rothman technique, Power's ratio, and basion-axial interval are unreliable because the radiologic landmarks in the occipito-cervical junction lack clarity in radiography.

Methods: One hundred four asymptomatic subjects were evaluated with lateral cervical radiographs in neutral, flexion and extension. They were stratified by age and included 52 young ( $20-29$ years) and 52 middle-aged adults ( $50-59$ years). The four radiographic reference points were posterior edge of hard palate (hard palate), posteroinferior corner of the most posterior upper molar tooth (molar), posteroinferior corner of the $\mathrm{C} 1$ anterior ring (posterior C1), and posteroinferior corner of the $\mathrm{C} 2$ vertebral body (posterior C2). The distance from posterior $\mathrm{C} 1$ and posterior $\mathrm{C} 2$ to the above anatomical landmarks was measured to calculate the range of motion (ROM) on dynamic radiographs. To determine the difference between the two age groups, unpaired $t$-tests were used. The statistical significance level was set at $p<0.05$.

Results: The ROM was $4.8 \pm 7.3 \mathrm{~mm}$ between the hard palate and the posterior $\mathrm{C} 1,9.9 \pm 10.2 \mathrm{~mm}$ between the hard palate and the posterior $\mathrm{C} 2,1.7 \pm 7.2 \mathrm{~mm}$ between the molar to the posterior $\mathrm{C} 1$, and $10.4 \pm 12.1 \mathrm{~mm}$ between the molar to the posterior $\mathrm{C} 2$. There was no statistically significant difference for the ROM between the young- and the middle-aged groups. The intra-observer reliability for new radiographic index was good. The inter-observer reliability for the ROM measured by the hard palate was low, but was better than that by the molar.

Conclusions: ROM measured by the hard palate might be a useful new radiographic index in cases of occipito-cervical instability.
\end{abstract}

Keywords: Occipito-cervical instability; Radiographic index; Range of motion

\section{Introduction}

Symptomatic atlanto-occipital instability requires the fu- sion of the atlanto-occipital joint. Reports have described the diagnostic tools for atlanto-occipital instability in patients with Down's syndrome or in healthy volunteers

\footnotetext{
Received May 20, 2015, Revised Jun 20, 2015, Accepted Jun 26, 2015

Corresponding author: Moon Soo Park

Department of Orthopaedic Surgery, Hallym University Sacred Heart Hospital, Hallym University College of Medicine,

22 Gwanpyeong-ro 170beon-gil, Dongan-gu, Anyang 14068, Korea

Tel: +82-31-380-6000, Fax: +82-31-380-6008, E-mail: amhangpark@gmail.com
} 
[1-7]. However, measurements of occipito-cervical translation using the Wiesel-Rothman technique, Power's ratio, and basion-axial interval are not reproducible [8]. Any of the widely utilized plain radiographic criteria for basilar invagination at the occipito-cervical junction are unreliable because the radiologic landmarks in the occipitocervical junction lack clarity in radiography [9].

We evaluated a new method to evaluate occipito-cervical instability using the hard palate and the most posterior upper molar tooth, which are the structures imbedded in the bony structure of the skull $(\mathrm{C} 0)$. We propose a new index for determining atlanto-occipital instability (C0-C1 instability) and occipito-cervical instability (C0-C2 instability).

\section{Materials and Methods}

\section{Patient recruitment}

This study was approved by the Institutional Review Board at the institution of the corresponding author (IRB number: 2014-I085). Seven hundred ninety one healthy asymptomatic adults who visited out clinic because of back pain underwent neutral and dynamic cervical radiographs from January 2009 to December 2011. They had no symptoms or signs of cervical degeneration or deformity. Cervical and lumbar spine evaluations were performed. Patients with previous history of cervical trauma or operations were excluded. We randomly selected people in their 20 s (20 to 29 years) and 50 s (50 to 59 years). The youngaged group contained 52 people (16 men, 36 women; mean age, $24.8 \pm 3.1$ years). The middle-aged group contained 52 people (14 men, 38 women; mean age, 53.4 \pm 2.9 years). The mean body mass index (BMI) of the youngaged group and middle-aged group was $22.8 \pm 3.6 \mathrm{~kg} / \mathrm{m}^{2}$ and $23.6 \pm 2.4 \mathrm{~kg} / \mathrm{m}^{2}$, respectively. Gender and mean BMI were not significantly different between the two groups $(p>0.05)$.

\section{Measurement of radiographic parameters}

All patients had cervical spine lateral radiographs in neutral, flexion, and extension. The images were obtained with the patients standing and looking straight ahead. Lateral radiographs were performed using standard radiographic techniques wherein the tube was centered on the C3-4 intervertebral disc. The radiographic film cassette was 72 inches from the tube and radiographs were taken without magnification. The digital X-ray images were obtained on the PACS II view system (Infinitt, Seoul, Korea), which was used to obtain measurements. We set four radiographic reference points: the posterior edge of the hard palate (hard palate), the posteroinferior corner of the most posterior upper molar tooth (molar), the posteroinferior corner of $\mathrm{C} 1$ anterior ring (posterior $\mathrm{C} 1$ ), and the posteroinferior corner of $\mathrm{C} 2$ vertebral body (posterior $\mathrm{C} 2$ ). We measured the distances from the posterior edge of the hard palate to the posteroinferior corner of the $\mathrm{C} 1$ anterior ring (hard palate-posterior $\mathrm{C} 1$ distance) to the posteroinferior corner of $\mathrm{C} 2$ vertebral body (hard palateposterior C2 distance) (Fig. 1, red lines). We also measured the distances from the posteroinferior corner of the most posterior upper molar tooth to the posteroinferior corner of the $\mathrm{C} 1$ anterior ring (molar-posterior C1 distance) and the posteroinferior corner of the $\mathrm{C} 2$ vertebral body (molar-posterior C2 distance) (Fig. 1, blue lines). Measurements were made on neutral, flexion, and extension lateral radiographs. The range of motion (ROM) was defined as the distance with extension minus the distance with flexion.

\section{Statistical methods}

Data were analyzed data using SPSS ver. 17.0 for Windows

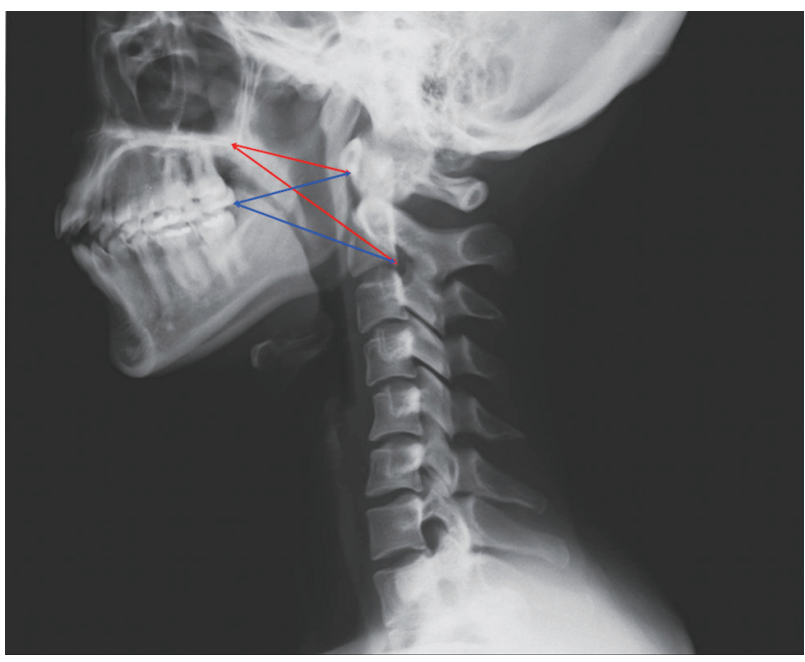

Fig. 1. Distances from the posterior edge of hard palate to the posteroinferior corner of $\mathrm{C} 1$ anterior ring (Hard palate, posterior C1 distance, red line), to posteroinferior corner of C2 vertebral body (Hard palate, posterior C2 distance, red line), and the distances from the posteroinferior corner of most posterior upper molar tooth to the posteroinferior corner of $\mathrm{C} 1$ anterior ring (Molar, posterior C1 distance, blue line) or to posteroinferior corner of $\mathrm{C} 2$ vertebral body (Molar, posterior C2 distance, blue line). 
Table 1. Distance by each radiologic landmarks (mm)

\begin{tabular}{llll} 
Distance & Neutral & Flexion & Extension \\
Hard palate-posterior C1 distance & $44.6 \pm 5.4$ & $44.9 \pm 5.0$ & $50.1 \pm 6.3$ \\
Hard palate-posterior C2 distance & $70.6 \pm 6.8$ & $68.7 \pm 6.0$ & $79.4 \pm 7.7$ \\
\hline Molar-posterior C1 distance & $45.1 \pm 7.7$ & $46.5 \pm 6.1$ & $48.7 \pm 7.7$ \\
Molar-posterior C2 distance & $62.0 \pm 6.5$ & $60.3 \pm 6.6$ & $72.2 \pm 8.5$ \\
\hline
\end{tabular}

Table 2. Range of motion by each radiologic landmarks according to age groups ( $\mathrm{mm}$ )

\begin{tabular}{lcccc} 
Range of motion (ROM) & Total & Young-aged & Middle-aged & $p$-value \\
Hard palate-posterior C1 ROM & $4.8 \pm 7.3$ & $3.5 \pm 4.5$ & $6.0 \pm 9.2$ & 0.085 \\
Hard palate-posterior C2 ROM & $9.9 \pm 10.2$ & $9.6 \pm 6.8$ & $10.1 \pm 12.8$ & 0.832 \\
Molar-posterior C1 ROM & $1.7 \pm 7.2$ & $1.7 \pm 4.2$ & $1.7 \pm 9.3$ & 0.987 \\
Molar-posterior C2 ROM & $10.4 \pm 12.1$ & $11.3 \pm 7.6$ & $9.5 \pm 15.3$ & 0.467 \\
\hline
\end{tabular}

Table 3. Range of motion (ROM) by each radiologic landmarks according to sex (mm)

\begin{tabular}{lcccc} 
Range of motion (ROM) & Total & Female & Male & $p$-value \\
Hard palate-posterior C1 ROM & $4.8 \pm 7.3$ & $5.2 \pm 8.1$ & $4.1 \pm 6.1$ & 0.437 \\
Hard palate-posterior C2 ROM & $9.9 \pm 10.2$ & $10.6 \pm 12.0$ & $8.8 \pm 7.0$ & 0.409 \\
Molar-posterior C1 ROM & $1.7 \pm 7.2$ & $2.4 \pm 8.6$ & $0.8 \pm 4.9$ & 0.299 \\
Molar-posterior C2 ROM & $10.4 \pm 12.1$ & $11.4 \pm 14.4$ & $8.9 \pm 7.8$ & 0.327 \\
\hline
\end{tabular}

(SPSS Inc., Chicago, IL, USA). Unpaired $t$-tests were used to determine differences between age groups and gender groups. Power analysis was performed using $\mathrm{G}^{\star}$ Power ver. 3.1.5 (Dusseldorf, Germany). Power was 0.95 for unpaired $t$-tests with the effect size of 0.8 . The sample size in each group should be more than 42 . The statistical significance level was set at $p<0.05$. The intra-observer and interobserver reliability were calculated using the reliability statistics by intraclass correlation (ICC) for the distance on the radiographs $[10,11]$. The ICC values were graded using previously described semiquantitative criteria: excellent for values in the 0.9-1.0 range, good for 0.7-0.89, fair/moderate for $0.50-0.69$, low for $0.25-0.49$, and poor for $0.0-0.24$.

\section{Results}

The hard palate-posterior $\mathrm{C} 1$ distance, hard palateposterior C2 distance, molar-posterior $\mathrm{C} 1$ distance, and molar-posterior $\mathrm{C} 1$ distance was $44.6 \pm 5.4 \mathrm{~mm}, 70.6 \pm 6.8$ $\mathrm{mm}, 45.1 \pm 7.7 \mathrm{~mm}$, and $62.0 \pm 6.5 \mathrm{~mm}$, respectively, in the neutral position (Table 1). These distances increased with extension (Table 1).

The ROM between the hard palate and the posterior $\mathrm{C} 1$, between the hard palate and the posterior $\mathrm{C} 2$, between the molar and the posterior $\mathrm{C} 1$, and between the molar and the posterior C2 was $4.8 \pm 7.3 \mathrm{~mm}$ (range, -43.3 to $21.5 \mathrm{~mm}$ ), $9.9 \pm 10.2 \mathrm{~mm}$ (range, -61.7 to $24.2 \mathrm{~mm}$ ), $1.7 \pm 7.2$ $\mathrm{mm}$ (range, -43.3 to $32.5 \mathrm{~mm}$ ), and $10.4 \pm 12.1 \mathrm{~mm}$ (range, -57.2 to $30.6 \mathrm{~mm}$ ), respectively, in the total study population (Table 2). The distances between the hard palate and the posterior $\mathrm{C} 1$, between the hard palate and the posterior $\mathrm{C} 2$, between the molar and the posterior $\mathrm{C} 1$, and between the molar and the posterior $\mathrm{C} 2$ decreased during extension and increased during flexion in some patients. The ROM measured at the atlanto-occipital joint according to the hard palate was $<45 \mathrm{~mm}$ and the ROM of the occipito-cervical joint (C0-C2 $\mathrm{ROM})$ according to the hard palate was $<65 \mathrm{~mm}$. There was no statistical difference for the ROM between the two age groups (Table 2). There was no statistical difference for the ROM by gender (Table 3).

The reliability statistics by ICC for ROM measured by the hard palate and the most posterior upper molar tooth 
Table 4. The reliability statistics by intraclass correlation for the range of motion based on the dynamic radiographs

\begin{tabular}{lcc} 
Range of motion (ROM) & Intra-observer & Inter-observer \\
Hard palate-posterior C1 ROM & 0.770 & 0.494 \\
Hard palate-posterior C2 ROM & 0.783 & 0.456 \\
Molar-posterior C1 ROM & 0.774 & 0.337 \\
Molar-posterior C2 ROM & 0.750 & 0.405 \\
\hline
\end{tabular}

Table 5. Literatures about the atlanto-occipital instability with dynamic radiographs

\begin{tabular}{|c|c|c|c|c|}
\hline Authors & Subjects & Number & Method & Limits (mm) \\
\hline Wiesel and Rothman [1] & Healthy adults/patients & 20 healthy/2 patients & Wiesel-Rothman technique & $>1$ \\
\hline Uno et al. [2] & Down syndrome & 75 & Wiesel-Rothman technique & range $1.0-9.0$ \\
\hline Tredwell et al. [3] & Down syndrome & 64 & $\begin{array}{l}\text { Anterior margin of condyle to } \\
\text { atlas anterior aspect sharp } \\
\text { contour }\end{array}$ & $>4$ \\
\hline Parfenchuck et al. [4] & Down syndrome & 199 & Power's ratio & $<0.55$ in extension \\
\hline El-Khoury et al. [5] & Down syndrome & 3 & Wackenheim line & - \\
\hline
\end{tabular}

was $0.750-0.783$ for the intra-observer reliability (Table 4). The inter-observer reliability for ROM measured by the hard palate was $0.456-0.494$, however that by the most posterior upper molar tooth was $0.337-0.405$ (Table 4 ).

\section{Discussion}

Previous studies have radiographically evaluated the occpito-cervical junction [1-7,12-14]. However, none of the widely utilized plain radiographic criteria for occipitocervical junction have proven to be reliable $[8,9]$. Here, we propose a new index for atlanto-occipital instability $(\mathrm{C} 0-$ $\mathrm{C} 1$ instability) and occipito-cervical instability $(\mathrm{C} 0-\mathrm{C} 2$ instability).

The ROM between the hard palate and the posterior $\mathrm{C} 1$, between the hard palate and the posterior $\mathrm{C} 2$, between the molar and the posterior $\mathrm{C} 1$, and between the molar and the posterior $\mathrm{C} 2$ was $4.8 \pm 7.3 \mathrm{~mm}, 9.9 \pm 10.2 \mathrm{~mm}, 1.7 \pm 7.2$ $\mathrm{mm}$, and $10.4 \pm 12.1 \mathrm{~mm}$, respectively. The ROM of the atlanto-occipital joint was $<45 \mathrm{~mm}$ and the ROM of the occipito-cervical joint ( $\mathrm{C} 0-\mathrm{C} 2 \mathrm{ROM})$ was $<65 \mathrm{~mm}$. The intra-observer reliability for new radiographic index was good. However, the inter-observer reliability was low. The inter-observer reliability for ROM measured by the hard palate was better than that by the most posterior upper molar tooth.

Previous studies have evaluated atlanto-occipital in- stability with dynamic radiographs (Table 5). Wiesel and Rothman [1] described a technique in which translational motion on dynamic radiographs as measured between the occiput and $\mathrm{C} 1$. The normal value for atlanto-occipital translational motion using the Wiesel-Rothman technique is $1 \mathrm{~mm}$ in healthy adults [1]. Uno et al. [2] also used the Wiesel-Rothman technique in 75 patients with Down's syndrome to evaluate the overall range of anteroposterior atlanto-occipital motion (mean $2.3 \mathrm{~mm}$; range, 1.0-9.0 $\mathrm{mm}$ ). The atlanto-occipital relationship was measured as the distance between the anterior margin of the condyles at the base of the skull and the sharp contour of the anterior aspect of the atlas in 64 patients with Down's syndrome [3]. In cases with atlanto-occipital instability, there was more than $4 \mathrm{~mm}$ of motion on dynamic radiographs. In a study of 199 patients with Down's syndrome, Parfenchuck et al. [4] found that those with posterior atlanto-occipital hypermobility had a Power's ratio under 0.55 in extension. Power's ratio is a ratio of the distance between the basion and the posterior arch of $\mathrm{C} 1$ to the distance between the opisthion and the anterior arch of $\mathrm{C} 1$. The ratio in normal individuals is $0.77 \pm 0.09$ [15]. El-Khoury et al. [5] defined atlanto-occipital instability using the relative position of the Wackenheim line based on the dynamic radiographs of three patients with Down's syndrome. In the current study, the ROM of the atlanto-occipital joint was $<45 \mathrm{~mm}$. This could be because the measuring methods in previ- 
Table 6. Literatures about the occipito-cervical instability (CO-C2 instability)

\begin{tabular}{|c|c|c|c|c|c|}
\hline Authors & Subjects & Number & Radiograph & Method & Limits (mm) \\
\hline Harris et al. [13] & $\begin{array}{l}\text { Healthy adults and } \\
\text { children }\end{array}$ & 400 adults/50 children & Neutral & Basion-axial interval & $>12$ \\
\hline Harris et al. [13] & Healthy adults & 25 & Dynamic & Basion-axial interval & range 0-10 \\
\hline Brockmeyer et al. [12] & Atlantal hemiring & 19 & Dynamic & $\begin{array}{l}\text { Clivus to C2 vertebral } \\
\text { body posterior border }\end{array}$ & $>6$ \\
\hline Wholey et al. [14] & $\begin{array}{l}\text { Healthy adults and } \\
\text { children }\end{array}$ & 480 adults/120 children & Neutral & Basion-dens interval & $>10$ \\
\hline Harris et al. [13] & Healthy adult & 400 & Neutral & Basion-dens interval & range $2-15$ \\
\hline El-Khoury et al. [5] & Healthy adolescents & 40 & Dynamic & Basion-dens interval & $\begin{array}{l}7.1 \text { (flexion) } \\
8.4 \text { (extension) }\end{array}$ \\
\hline
\end{tabular}

Basion-axial interval, the distance between the basion and a line drawn along the posterior edge of the vertebral body of the axis; Basion-dens interval, the distance between the basion and the odontoid tip.

ous studies were different from those in the current study; some of the measurements showed the distances from the vertical axis only $[1,2,5]$, but not the horizontal and vertical axes, and evaluated patients with Down's syndrome (Table 5) [2-5].

Studies have also evaluated occipito-cervical instability (C0-C2 instability) with basion-axial interval (Table 6). The basion-axial interval is the distance between the basion and a line drawn along the posterior edge of the vertebral body of the axis [13]. Based on the neutral cervical radiographs, Harris et al. [13] found that $\mathrm{C} 0-\mathrm{C} 2$ instability was defined as $>12 \mathrm{~mm}$ of the basion-axial interval in 400 healthy adults and 50 children. Based on the dynamic cervical radiographs, the authors found that the excursion of basion-axial interval in 25 healthy adults was $0-10 \mathrm{~mm}$ [13]. Brockmeyer et al. [12] reported that $\mathrm{C} 0-\mathrm{C} 2$ instability was defined when the distance from clivus to $\mathrm{C} 2$ vertebral body posterior border exceeded $6 \mathrm{~mm}$ in dynamic radiographs of patients with atlantal hemiring.

Wholey et al. [14] introduced the basion-dens interval for the occipito-cervical instability ( $\mathrm{C} 0-\mathrm{C} 2$ instability) in 1956 (Table 6). The basion-dens interval is defined as the distance between the basion and the odontoid tip. The authors suggested that basion-dens interval should be maintained under $10 \mathrm{~mm}$ in the neutral radiographs of normal young child. Harris et al. [13] found that basiondens interval in neutral radiographs of 400 healthy adults was $2-15 \mathrm{~mm}$. The $95 \%$ accuracy range was $11.8 \mathrm{~mm}$. ElKhoury et al. [5] reported that the average basion-dens interval in the dynamic radiographs of 40 healthy adults was $7.1 \mathrm{~mm}$ in flexion and $8.4 \mathrm{~mm}$ in extension. Contrarily, in the current study the ROM of the occipito-cervical joint
(C0-C2 ROM) was maintained as $<65 \mathrm{~mm}$. This could be because the prior studies evaluated the instability based on neutral radiography $[13,14]$, and because the authors included study populations that differed from that of the current study including healthy children, healthy adolescents, and patients with atlantal hemiring, and because they used the distances from the vertical axis only, not the horizontal and vertical axes (Table 6) [5,12-14].

In contrast to the angular changes seen using Cobb's angles during flexion-extension, the distances between the hard palate and the posterior $\mathrm{C} 1$, between the hard palate and the posterior $\mathrm{C} 2$, between the molar and the posterior $\mathrm{C} 1$, and between the molar and the posterior $\mathrm{C} 2$ increased during flexion and decreased during extension in some patients in the current study. In contrast, these angular changes decreased during flexion and increased during extension in other patients. This might be because coupled translation accompanies the motion of flexion and extension at atlanto-occipital joints. At the atlantooccipital joint, anterior-posterior translation commonly occurs during flexion-extension due to the oval shapes of the occipital condyles and lateral masses [16].

As with any study, the present investigation has several limitations. First, the inter-observer reliability was low. Second, although all of the subjects were positioned in the exact same manner, we cannot with certainty assume that our data is completely accurate. Third, it is a retrospective study. Perhaps a prospective study would yield different results, although as a radiographic study we are not sure that there would be substantial differences. Fourth, we need to prove that new radiographic index is useful in the symptomatic patients with $\mathrm{C} 0-\mathrm{C} 1$ and $\mathrm{C} 0-\mathrm{C} 2$ instability. 
Fifth, the intra-observer reliability for new radiographic index was good, and the inter-observer reliability was low. However, the inter-observer reliability for ROM measured by the hard palate was better than that by the most posterior upper molar tooth. The hard palate is more reliable because the left and right most posterior upper molar teeth are not perfectly superimposed on the radiograph, many do not have molar teeth, some still have their wisdom teeth and some have malformed or crooked molar teeth.

Despite these shortcomings, to our knowledge, this is the first report proposing a new radiographic index for the occipito-cervical junction that could be used as a simple guide in clinical practice.

\section{Conclusions}

Utilizing our proposed measurement parameters of the hard palate confirmed the ROM of atlanto-occipital joint maintained $<45 \mathrm{~mm}$ and the ROM of occipito-cervical joint (C0-C2 ROM) maintained $<65 \mathrm{~mm}$. All ROM maintained similarly regardless of sex and in young and middle-aged group.

\section{Conflict of Interest}

No potential conflict of interest relevant to this article was reported.

\section{References}

1. Wiesel SW, Rothman RH. Occipitoatlantal hypermobility. Spine (Phila Pa 1976) 1979;4:187-91.

2. Uno K, Kataoka O, Shiba R. Occipitoatlantal and occipitoaxial hypermobility in Down syndrome. Spine (Phila Pa 1976) 1996;21:1430-4.

3. Tredwell SJ, Newman DE, Lockitch G. Instability of the upper cervical spine in Down syndrome. J Pediatr Orthop 1990;10:602-6.

4. Parfenchuck TA, Bertrand SL, Powers MJ, Drvaric DM, Pueschel SM, Roberts JM. Posterior occipitoatlantal hypermobility in Down syndrome: an analysis of 199 patients. J Pediatr Orthop 1994;14:304-8.

5. El-Khoury GY, Clark CR, Dietz FR, Harre RG, Tozzi
JE, Kathol MH. Posterior atlantooccipital subluxation in Down syndrome. Radiology 1986;159:507-9.

6. Menezes AH, Ryken TC. Craniovertebral abnormalities in Down's syndrome. Pediatr Neurosurg 1992;18: 24-33.

7. Browd S, Healy LJ, Dobie G, et al. Morphometric and qualitative analysis of congenital occipitocervical instability in children: implications for patients with Down syndrome. J Neurosurg 2006;105:50-4.

8. Karol LA, Sheffield EG, Crawford K, Moody MK, Browne RH. Reproducibility in the measurement of atlanto-occipital instability in children with Down syndrome. Spine (Phila Pa 1976) 1996;21:2463-7.

9. Riew KD, Hilibrand AS, Palumbo MA, Sethi N, Bohlman HH. Diagnosing basilar invagination in the rheumatoid patient: the reliability of radiographic criteria. J Bone Joint Surg Am 2001;83:194-200.

10. Kepler CK, Sharma AK, Huang RC, et al. Indirect foraminal decompression after lateral transpsoas interbody fusion. J Neurosurg Spine 2012;16:329-33.

11. Koch GG. Intraclass correlation coefficient. In: Kotz S, Johnson NL, Read CB, editors. Encyclopedia of statistical sciences. New York: Wiley; 1982. p.213-7.

12. Brockmeyer DL, Brockmeyer MM, Bragg T. Atlantal hemi-rings and craniocervical instability: identification, clinical characteristics, and management. J Neurosurg Pediatr 2011;8:357-62.

13. Harris JH Jr, Carson GC, Wagner LK. Radiologic diagnosis of traumatic occipitovertebral dissociation: 1. Normal occipitovertebral relationships on lateral radiographs of supine subjects. AJR Am J Roentgenol 1994;162:881-6.

14. Wholey MH, Bruwer AJ, Baker HL Jr. The lateral roentgenogram of the neck; with comments on the atlanto-odontoid-basion relationship. Radiology 1958;71:350-6.

15. Powers B, Miller MD, Kramer RS, Martinez S, Gehweiler JA Jr. Traumatic anterior atlanto-occipital dislocation. Neurosurgery 1979;4:12-7.

16. Radcliff KE, Hussain MM, Moldavsky M, et al. In vitro biomechanics of the craniocervical junction-a sequential sectioning of its stabilizing structures. Spine J 2015;15:1618-28. 